\title{
125I Low Dose Rate Radiation Increases Radiotherapy Sensitivity on Esophageal Cancer Cells via Multiple Mechanisms
}

Hui Xue

Peking University Third Hospital, Beijing

Lifa Du

Peking University Third Hospital, Beijing

Chunxiao Li

Peking University Third Hospital, Beijing

Hao Wang

Peking University Third Hospital, Beijing

\section{Lixiang Xue}

Peking University Third Hospital, Beijing

Junjie Wang

Peking University Third Hospital, Beijing

Ping Jiang ( $\nabla$ driangping@qq.com )

Peking University Third Hospital, Beijing https://orcid.org/0000-0001-8820-489X

\section{Research}

Keywords: 125 I radioactive seeds continuous low dose radiation, cell cycle, apoptosis, autophagy, esophageal cancer

Posted Date: October 16th, 2020

DOI: https://doi.org/10.21203/rs.3.rs-92223/v1

License: (9) This work is licensed under a Creative Commons Attribution 4.0 International License. Read Full License 


\section{Abstract}

Objective: To determine the biological effectiveness of iodine $125\left({ }^{125} \mathrm{I}\right)$ radioactive seeds continuous low dose rate radiation on the human esophageal cancer cell line KYSE150 compare to single dose radiation and explore its potential cellular mechanisms.

Method: Three groups of KYSE150 cells were explored: control group, single dose irradiation (SDR) and ${ }^{125}$ I seeds continuous low dose rate irradiation group ( ${ }^{125}$ I-CLDR). Dose-survival curves was obtained by colony formation assay. MTT proliferation assay was used to measure KYSE150 cell vibility. KYSE150 cell apoptosis was analysis by Annexin V-FITC/PI staining, meanwhile, cell cycle analysis was performed by flow cytometry. Bim, $\mathrm{Bcl}-2$, caspase-3 and cleaved caspase-3 protein expression were measured by western blotting to vertify the apoptosis level, as well as CyclinB1 protein expression which values the fuction of $\mathrm{G} 2 / \mathrm{M}$ check point.Cell morphology changes were observated under phase contrast microscope. Endoplasmic reticulum stress (ER stress) was measured by GPR78/Bip1 and PERK changes in gene expression, which was detected by Real-time PCR. Reactive oxygen species(ROS) changes and mitochondria were meaured by flow cytometry. ATP detection kit was used to measured ATP level afer two modes of irradiation. DNA-Pks, Ku70 and Ku80 expression were measured by western blotting to represent the damage of DNA damage and repair capabilities. Acridine orange (AO) staining was used to detect level of autophagy and quantitative was measured by flow cytometry. LC3-II, ATG5, Beclin1, p-Akt, p-mTOR, mTOR and p-S6 proteins expression were detectd by western blotting.

Results: KYSE150 cells were more radiosensitive to 125I-CLDR than SDR. Two modes of irradiation could both inhibit the proliferation vability of KYSE150 cells, while the inhibition effects in 125I-CLDR was significantly stronger compared with SDR. Compared with SDR, 125I-CLDR showed more proportions of the early and late apoptosis rate as well as cells at G2/M phase. Apoptosis related proteins, such as Bim, caspase-3 and cleaved caspase-3, were elevated, while CyclinB1 expression was decreased in 125ICLDR group. Cells became bigger and grainy in 125I-CLDR group, meanwhile, ROS levels and ER stress signal was elevated except ATP concentration. Cells stained by AO was anaysised by flow cytometry, results showed that red: green ratio in 125I-CLDR group increased as well as LC3 and ATG5 protein expression detected by western blotting. p-Akt, p-mTOR and p-S6 protein expression were decreased to some extent in 125I-CLDR group cells, while total mTOR protein showed no significant changes.

Conclusion: Our results confirmed that ${ }^{125}$ I-CLDR could strong inhibite KYSE150 cancer cells. Furthermore, we revealed that cell cycle arrest and apoptosis were not the only fate after 125I-CLDR, autophagy might be another important choice which mTOR pathway might be involed in. These findings can support the application of esophageal stent loaded with $125 \mathrm{I}$ seeds in clinic.

\section{Introduction}

Esophageal cancer is one of the most aggressive gastrointestinal cancers and it remains a global health concern with a dismal prognosis and an estimated 5-year survival rate of approximately $10-15 \%[1,2]$. 
Dysphagia is one of the main clinical symptoms and prognostic factors[3]. Available palliative options for dysphagia are esophageal stent placement and radiotherapy. In general, intraluminal brachytherapy of high dose rate can relieve dysphagia and improve the quality of patients' life, but other severe symptoms such as esophageal fistula, perforation and bleeding. Self-expanding esophageal stent can effectively relieve the patients' clinical symptoms, however, it is no therapeutic effect, esophageal recurrence and narrowness cannot be avoided [3,4]. Due to limited availability of intra-luminal brachytherapy and self-expanding esophageal stent in clinical practice, ${ }^{125}$ I radioactive seeds loaded with esophageal stent exhibits the advantages of both options and the significant clinical effect [5]. Although there are several reports on the biological effects of continuous low dose rate radiation, the different effectiveness of variant dose rate irradiation on the human esophageal cancer cell lines and the relevant cellular mechanisms is still largely unidentified. The aim of the present study is to determine the direct biological effectiveness of single dose radiation (SDR) and ${ }^{125}$ I continuous low dose rate radiation ( ${ }^{125}$ CLDR) on human esophageal cancer cell line KYSE150 in vitro, and we also aimed to unravel the underlying cellular and molecular mechanisms behind distinct modes of irradiation.

The sensitivity of cancer to radiotherapy is affected by many factors. Activation of DNA damage response (DDR) pathways is considered an important factor affecting resistance to radiotherapy[6]. Loss of cell-cycle checkpoint responses may result in increased sensitivity, particularly if the checkpoint controls the $\mathrm{G} 2$ transition. Overexpression of cyclin $\mathrm{D}$, which shortens the duration of the $\mathrm{G} 1$ transition, is associated with radiation resistance, perhaps by inhibiting apoptosis[7]. ROS are the effector molecules of radiation, and increase of ROS production is known to enhance radioresponse[8]. The role of autophagy in radiotherapy sensitivety has been paid more and more attention. However, the role of autophagy in radiotherapy sensitivity in tumor cells remains controversial[9]. In the radiotherapy of esophageal cancer studies have shown that autophagy promotes the survival of esophageal cancer to resist tumor radiation therapy [10], and recent studies have found that autophagy can promote the death of cancer cells and increase their radiotherapy sensitivity [11]. What's more, although the common upstream signaling pathways have been found which is linked to apoptosis and autophagy [12], the potential regulatory mechanisms have not been clearly understood.

Therefore, in the present study, different biological effects of single dose radiation (SDR) and ${ }^{125}$ I continuous low dose rate radiation ( ${ }^{125}$ I-CLDR) on human esophageal cancer cell line KYSE150 were explored in vitro. Moreover, we analyzed the inhibitory mechanisms of ${ }^{125}$ I-CLDR to cancer cell proliferation, including analysis of the cell cycle, apoptosis, ER stress, mitochondrial function and autophagy in human esophageal cancer cell line KYSE150 in vitro. Given the established roles of the AktmTOR signaling pathway and ROS as some of the early changes linked to apoptosis and autophagy, we further examined their potential involvement in the inhibitory effects induced by ${ }^{125}$ I-CLDR. This work highlights the great potential clinical applications of ${ }^{125}$-CLDR in the treatment of esophageal cancer.

\section{Materials And Methods}




\section{Cell culture}

The human esophageal cancer cell lines KYSE150 was obtained from Chines academy of medical sciences Tumor Cell Bank (Beijing, China) and cultured in Roswell Park Memorial Institute (RPMI 1640, HyClone) containing $10 \%$ fetal bovine serum (FBS) (HyClone), 10 units $/ \mathrm{mL}$ penicillin, and $10 \mathrm{mg} / \mathrm{mL}$ streptomycin and maintained in humidified incubators at $37^{\circ} \mathrm{C}$ and $5 \% \mathrm{CO}_{2}$.

\section{$X$-ray and ${ }^{125}$ I seeds irradiation}

The irradiation method was performed as previously described [13]. Briefly, Liner accelerators producing 6 MV X-ray beams were provided by the 306th Hospital of the People's Liberation Army (Beijing, China). The dose rate was $400 \mathrm{cGy} / \mathrm{min}$ and the source-to-skin distance (SSD) was $100 \mathrm{~cm}$. The surface of the culture dishes was covered by $1.5 \mathrm{~cm}$ thickness packing materials. Radiation dose were 0, 2, 4, 6 and 8Gy. An inhouse model for in vitro iodine- 125 seed irradiation was developed for this study. The activity of the single seed used in this study was $92.5 \mathrm{MBq}(2.5 \mathrm{mCi})$, which translates to an initial dose rate of 2.77 $\mathrm{cGy} / \mathrm{h}$ to model cells. The exposure times for delivering doses of 2, 4, 6 and 8Gy were 73.7, 154.6, 245.8 and 345.1 hours (i.e., $3.07,6.44,10.24$ and 14.38 days), respectively $[14,15]$. The absorbed dose was equivalent to SDR treatment.

\section{Colony formation assay}

Colony formation assay was used to detect the radiation sensitivity of KYSE150 cells after different modes irradiation. Different numbers of cells were plated into 6-well plates depending on the doses of radiation: 0Gy (100), 2Gy (200), 4Gy (800), 6Gy (1600) and 8Gy (3200). And, the transfected cells were subjected to radiation at the above indicated doses. Then, the cells were cultured for 14 days to form colonies, and next, stained with methylene blue. Those colonies ( $\geq 50$ cells) were then counted. The experimental data was fitted to the single-hit multi-target model to make the survival curves of different groups.

\section{MTT proliferation assay}

MTT proliferation assay was used to measure KYSE150 cell viability after two modes irradiation on the

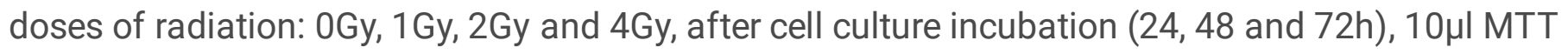
(dissolved in RPMI-1640, $5 \mathrm{mg} / \mathrm{mL}$ ) was added to well, and plates were further incubated at $37{ }^{\circ} \mathrm{C}$ for $4 \mathrm{~h}$. Finally, the purple formazan crystals were dissolved by adding $100 \mu \mathrm{l}$ acid-isopropanol $(0.04 \mathrm{~N} \mathrm{HCl}$ in isopropanol) into each well. Absorbance was then measured at $550 \mathrm{~nm}$ against reference wavelength of $630 \mathrm{~nm}$ and stimulation index (SI) was determined. Te $\mathrm{SI}$ is expressed with average OD value in the test group divided by average OD value in negative controls. Cell proliferation activity $(\%)=($ each dose point) Absorbance value/(0 Gy) Absorbance value.

\section{Cell cycle analysis}


Cells after two modes irradiation on the doses of 4Gy for 72h, were harvested and washed with PBS for 3 times and then fixed in pre-cooled $70 \%$ ethanol overnight at $-20^{\circ} \mathrm{C}$. Fixed cells were then pelleted through centrifugation, washed with PBS and incubated with $20 \mathrm{ug} / \mathrm{mL}$ RNase A (Sigma-Aldrich) in PBS at $37^{\circ} \mathrm{C}$ for 30 min. Cells were then analyzed on a BD FACS Calibur (BD bioscience, San Jose, CA, USA) after stained with $10 \mathrm{mg} / \mathrm{ml} \mathrm{PI} \mathrm{(Sigma-Aldrich)} \mathrm{in} 0.1 \%$ Triton X-100. Cell cycle distribution was analyzed with the ModFit LT software (Verity Software House, Topsham, ME).

\section{Cellular apoptosis assay}

Cellular apoptosis was evaluated by FITC-conjugated Annexin V/propidium iodide (PI) staining followed by flow cytometry analysis.

\section{Cell morphology observation}

Cell morphology changes were observed under phase contrast microscope (Olympus IX71, Japan) after two modes of irradiation and photographed (magnification, $\times 800$ ), which was analyzed by flow cytometry.

\section{Detection of intracellular reactive oxygen species (ROS)}

ROS labeled by DCFH-DA probes was measured by flow cytometry in FL1 channel, at the meantime, mitochondria was detected by flow cytometry in FL1 channel and fluorescence microscope after MitoTracker Green fluorescent probe stained.

\section{Detection of ATP}

ATP detection kit was used to measured ATP level after two modes of irradiation, the detail was followed by protocol. ATP concentration was standardized to $\mathrm{nmol} / \mathrm{mg}$ after BCA protein

\section{RT-qPCR assay of endoplasmic reticulum stress (ER stress)}

ER stress was measured by GPR78/Bip1 and PERK changes in gene expression, which was detected by Real-time PCR. According to the product manual, TRIzol reagent (Invitrogen) was applied to isolate total RNA from the cell samples. Then, NanoDrop (Thermo Fisher Scientific, 2000C) was used to measure the concentration of RNAs. Reverse transcription was performed according to standard protocols using a RevertAid $^{\text {TM }}$ II First Strand cDNA synthesis Kit (Thermo Fisher Scientific Inc., Waltham, MA USA). For detection of mRNA, Talent qPCR PreMix (SYBR Green) (TIANGEN, China) was used to detect the mRNA expression. GAPDH was amplified as an internal standard. The primers for PCR were as follows: 


\begin{tabular}{|ll|}
\hline GPR78/Bip1-F & 5'-GAACGTCTGATTGGCGATGC-3' \\
\hline GPR78/Bip1-R & 5'-GAGTCGAGCCACCAACAAGA-3' \\
\hline PERK-F & 5'-GAACCAGACGATGAGACAGAG-3' \\
\hline PERK-R & 5'-GGATGACACCAAGGAACCG -3' \\
\hline GAPDH-F & 5'-ATCTCTGCCCCCTCTGCTGA-3' \\
\hline GAPDH-R & 5'-GATGACCTTGCCCACAGCCT-3' \\
\hline
\end{tabular}

\section{Acridine orange $(\mathrm{AO})$ staining was used to detect autophagy}

AO staining was used to detect level of autophagy in KYSE150 cells and quantitative was measured by flow cytometry with red: green signal intensity ratio, which was performed as previously described[16]. AO solution with final concentration of $1 \mathrm{~g} / \mathrm{ml}$ was used to stain adherent cells at $37^{\circ} \mathrm{C}$ for $15 \mathrm{~min}$, then the buffer solution without phenol red was used to rinse cells for 3 times. Cells was digested with trypin and resuspended in $5 \%$ serum PBS, cell concentration was adjusted and detected by flow cytometry. The excitation light was $488 \mathrm{~nm}$, and the emission light of green (510-530nm) and red (640-650nm) corresponded to FL1 and FL3 channels in flow cytometry (Beckman Coulter Epics XL, USA), respectively. The ratio of FL3 to FL1 represented the level of autophagy.

\section{Western blot and antibodies}

The whole-cell lysate was extracted with RIPA lysis buffer (50mM Tris [pH 7.4], $150 \mathrm{mM} \mathrm{NaCl}, 1 \%$ Triton X$100,1 \%$ sodium deoxycholate, $0.1 \%$ SDS, sodium orthovanadate, sodium fluoride, EDTA, from Beyotime Company). Then BCA assay was used to measure the protein concentration. Afterwards, $30 \mu \mathrm{g}$ protein was loaded onto sodium dodecyl sulfate-polyacrylamide gel (SDS-PAGE) to separate. After that, proteins on the gels were transferred to polyvinylidene fluoride (PVDF, 0.22um, Millipore Company) membrane, and the transferring time was based on the protein molecular weight. Then, the membranes were incubated with primary antibodies (at the appropriate dilution according to the supplementary table 6) in $6 \mathrm{ml}$ primary antibody dilution buffer with gentle agitation overnight at $4^{\circ} \mathrm{C}$. Followed that, the membranes were incubated with Anti-rabbit/mouse IgG, HRP-linked antibody (1:2000, Beyotime Company) in $10 \mathrm{ml}$ secondary antibody dilution buffer with gentle agitation for 2 hours at room temperature. After washing three times for 5 min each with $15 \mathrm{ml}$ of Tris Buffered Saline with Tween® 20 (TBST), the membranes were exposed to get the blot images with 1X SignalFire ${ }^{\mathrm{TM}}$ ECL Reagent (Millipore Company). anti-caspase-3, anti-Bim, anti-AKT, anti-mTOR, anti-p-S6, anti-p-mTOR, anti-ATG5 , anti-Beclin1, anti-DNA-PKcs, anti-Ku80 and anti-Ku70 antibodies were obtained from Cell Signling Technology, Inc (Cell Signaling Technology, MA, USA). Anti-CyclinB1 and anti-Bcl-2 antibodies were obtained from Abcam, Inc (Abcam,USA). Anti-LC3 were obtained from MEDICAL\&BIOLOGICAL LABORATIORIES CO, LTD, Inc (MEDICAL\&BIOLOGICAL LABORATIORIES CO, LTD, Japan). 


\section{Statistics analysis}

Each experiment was performed $\geq 3$ times and values were expressed as mean \pm S.D. Data analysis was done using the GraphPad Prism. The experimental data were analyzed using SPSS software 16.0 (SPSS, Inc., Chicago, IL, USA). Statistical significance among different groups was measured by Student's t test or one-way analysis of variance. A P-value $<0.05$ was considered to represent a statistically significant difference.

\section{Results}

\section{KYSE150 cells were more radiosensitive to ${ }^{125}$ I-CLDR than SDR.}

Two modes of irradiation could both inhibit the proliferation viability of KYSE150 cells, while the inhibition effects in ${ }^{125}$-CLDR was more significantly compared with SDR. After KYSE150 cells were irradiated with 1, 2, 4, 6 and $8 \mathrm{~Gy}$, the survival cells in SDR group and ${ }^{125}$ I-CLDR group gradually decreased with the increase of radiation dose, and the survival cells of ${ }^{125}$-CLDR group at each dose point were lower than SDR group, with statistically significant difference (Fig. 1a). MTT method was used to detect cell activity of KYSE150 cells after different irradiation, and it was found that the cell activity gradually decreased with the increase of time (Fig. 1b) and dose (Fig. 1c), and the toxic effect of ${ }^{125}$ CLDR on cells was more significant in ${ }^{125}$ I-CLDR group (Fig. 1b-c).

\section{Compared with SDR, more proportions of the early and late apoptosis rate were shown as well as cells at G2/M phase in ${ }^{125}$ I-CLDR group.}

To observe the death of cells after irradiation after placenta blue staining and it is found that the percentage of positive cells in ${ }^{125}$ I - CLDR group was obviously higher than in SDR group (Fig. 2a). To detect cell apoptosis after irradiation by flow cytometry instrument and it is found that apoptosis ratio was increased significantly in ${ }^{125} \mathrm{I}$-CLDR group (Fig. 2b), the difference was significant statistically (Fig. 2C) and the difference was dose dependence (Fig. 2d). Detection of the expression of apoptosisrelated proteins by Western blot and it revealed that the expression of apoptosis-precursor protein Bim and anti-apoptotic protein bcl-2 were all increased, at the same time, the expression of caspase-3, a direct indicator of apoptosis, was up-regulated in both group, and the expression of them in ${ }^{125}$ I-CLDR group was higher than in SDR group(Fig. 2e). After irradiation, cell cycle of KYSE150 cells was detected by flow cytometer (Fig. 2f), and it was found that the ratio of G2/M phase increased, and the difference was more

significant in ${ }^{125} \mathrm{I}$-CLDR group (Fig. 2g). Furthermore, we found that the expression of CylinB1 which is to regulation of G2/M point is decreased in ${ }^{125}$ - CLDR group, while it is increased in the SDR group (Fig. 2h).

Cells was larger and grainy in ${ }^{125} \mathrm{I}-$ CLDR group. 
Cell morphology in each group after irradiation were observed by phase contrast microscope. It was found that the cells were significantly larger and the vacuoles as well as granules increased after ${ }^{125}$ CLDR irradiation (Fig. 3a). The FS and SS were detected by flow cytometry after irradiation, the results showed that the volume and granulation of cells increased significantly after irradiation (Fig. 3b), and the difference was statistically significant in ${ }^{125}$ I-CLDR group (Fig. 3c-d).

\section{ER stress signal was elevated, and ATP concentration was decreased in ${ }^{125} \mathrm{I}$-CLDR group.}

After irradiation, the cells was loaded with Mito-Tracker Green (mitochondrial Green fluorescent probe) and mitochondrial fluorescence intensity was observed by fluorescence microscope (Fig. 4a). The intensity of mitochondrial fluorescence was analyzed quantitatively by flow cytometry (Fig. 4b). The intracellular ATP concentration was reduced significantly after irradiation in ${ }^{125}$ I-CLDR group (Fig. 4C). Analysis of the expression of ER stress marker GRP78/Bip1 and downstream activation pathway molecule PERK by Real-time PCR and it was found that the expression of them were up-regulated significantly at 3,6 and $12 \mathrm{~h}$ in ${ }^{125}$ I-CLDR group (Fig. $4 \mathrm{~d}-\mathrm{e}$ ).

\section{ROS levels was elevated, and DNA damage was aggravated in ${ }^{125}$ I-CLDR group.}

KYSE150 cells was loaded with DCFH-DA probe after irradiation and the ROS level of KYSE150 cells was analyzed by flow cytometry, it is found that ROS was significantly increased after irradiation in both two group, but the ROS level has increased dramatically in the ${ }^{125}$ I-CLDR group though it is declining with the increase of time (Fig. 5a), the difference was significant statistically (Fig. 5b). The expression levels of DNA-pks, Ku70 and Ku80, which is the components of DNA repair protein complex, were detected by Western blot and it showed the expression of DNA-pks and Ku70 was significantly down-regulated, while there was no significant difference in Ku80 in the ${ }^{125}$ I-CLDR group (Fig. 5c).

\section{Activation of Akt-mTOR signaling pathways was decreased, and autophagy was increased in ${ }^{125}$ I-CLDR group.}

Acridine orange (AO) staining can be used to assess the level of intracellular autophagy [17]. Detection of the red-green signal ratio by flow cytometry and it showed that the proportion was up-regulated after irradiation, and the increase was more obvious in the ${ }^{125}$ I-CLDR (Fig. 6a). Detection of autophagy-related protein expression by Western blot and it showed that autophagy related proteins LC3-II and ATG5 were significantly up-regulated in the ${ }^{125}$ I-CLDR, while Beclin1 was not changed significantly (Fig. 6c). Detection of the expression of Akt-mTOR pathway by Western blot and it showed that the expression of pAkt, p-mtor and p-S6 were significantly down-regulated, especially at $48 \mathrm{~h}$ after irradiation, while the expression of total mTOR was not changed significantly in ${ }^{125}$ I-CLDR group (Fig. 6b).

\section{Discussion}


${ }^{125}$ I seed-loaded stent placement has served as an effective palliation for malignant esophageal strictures in China[18]. Our results showed that two modes of irradiation could both inhibit the proliferation viability of KYSE150 cells, while the inhibition effects in ${ }^{125}$-CLDR were significantly stronger compared with SDR.

Cell cycle deregulation is one of the major hallmarks of cancer cells. The induction of cell cycle arrest may be an effective strategy to control the aberrant proliferation of cancer cells[19]. Our results demonstrated that ${ }^{125}$ I radioactive seeds continuous low dose rate radiation causes the $G_{2} / M$ phase arrest of KYSE150 cells which were associated with a decrease of cyclin B1. Cyclin B1 is present in the nucleus during the $G_{2} / M$ phase, where it participates in the initiation and completion of mitosis. Lack of cyclin B1 causes lengthening of G2/M phases [20]. Thus, our results indicate that ${ }^{125}$ I radioactive seeds continuous low dose rate radiation may modulate the expression of cell cycle-related proteins to induce $\mathrm{G}_{2} / \mathrm{M}$ phase arrest and inhibit the proliferation of KYSE150 cells consequently. However, decreased cyclin B1 expression is not necessarily linked to growth arrest. Thus, the sophisticated mechanism underlying the ${ }^{125}$ I radioactive seeds continuous low dose rate radiation is still to be explored. On the other hand, autophagy defect also could lead to cell cycle arrest in G2/M phase, resulting in prolonged $M$ phase and growth arrest [21]. Our results demonstrated that ${ }^{125}$ I radioactive seeds continuous low dose rate radiation increases autophagy, which may also lead to cell cycle arrest in the G2/M phase and increases radiosensitivity.

Apoptosis is a cell death mechanism and can be activated by compounds targeting extrinsic and intrinsic (mitochondrial) pathways[22]. The extrinsic pathway is related to activation of cell surface death receptors such as FasL and TNF-R. Alternatively, the mitochondria-mediated intrinsic pathway may be initiated by many stress conditions. For the mitochondria-mediated intrinsic pathway, the release of cytochrome $\mathrm{C}$ from the mitochondrial membrane into the cytosol is fundamental to apoptosome formation and activation of the caspase family $[23,25]$. Members of the Bcl 2 family also play a significant role in apoptosis by controlling mitochondrial membrane permeability and facilitating the passage of cytochrome $\mathrm{C}$ [26]. We found that ${ }^{125}$ I radioactive seeds continuous low dose rate radiation induced an increase in $\mathrm{Bcl} 2$ levels, and further provoked sharp increase of Bim and the consequent release of cytochrome $\mathrm{C}$ into the cytosol. Based on our results, an imbalance of Bim and $\mathrm{Bcl} 2$ proteins after treatment with ${ }^{125} \mathrm{I}$ radioactive seeds continuous low dose rate radiation may lead to the resulting in the release of cytochrome $C$ from the mitochondria into the cytosol to trigger caspase-3 activation, ultimately causing apoptosis in KYSE150 cells. Nevertheless, these findings do not rule out the possibility that activation of extrinsic pathways may also be involved in ${ }^{125} \mathrm{I}$ radioactive seeds continuous low dose rate radiation-induced cell apoptosis, since we did not explore the effect on cell surface death receptors.

Autophagy, an important and evolutionarily conserved mechanism for maintaining cellular homeostasis, is another antiproliferation pathway that plays an essential role in determining cellular fate. Recently, studies demonstrated that apoptosis can induce autophagy by regulation of autophagy proteins as well as the formation of autophagosomes [12]. Our results confirm these findings, given that ${ }^{125}$ I radioactive 
seeds continuous low dose rate radiation promoted remarkable levels of autophagy activity as evidenced by the accumulation of autophagy-related proteins, such as LC3-II and ATG5. In mammalian cells, apoptosis and autophagy are regulated by several important intracellular signaling pathways, including the Akt-mTOR signaling pathway [27-30]. ${ }^{125}$ I radioactive seeds continuous low dose rate radiation caused significant inhibition of Akt-mTOR and S6 phosphorylation. The Akt-mTOR signaling proteins exist in a phosphorylated form and suppress apoptosis and autophagy under normal conditions; however, when the levels of phosphorylated Akt-mTOR are downregulated, apoptosis and autophagy are triggered [31, 32], indicating that downregulated of this pathway might underlie the ${ }^{125}$ I radioactive seeds continuous low dose rate radiation induced excessive apoptosis and autophagy. In addition, ${ }^{125}$-CLDR could also strong trigger caspase-3 activation, causing decreased esophageal cancer cells Akt-mTOR pathway signaling, which in turn increases autophagy. Apoptosis and autophagy are both highly regulated biological processes that may take place independently; however, in most circumstances, both processes are tightly connected by some common upstream signaling components. For example, there are common upstream signaling pathways between autophagy and apoptosis induced by ER stress, including ATF4, IRE1a, ATF6 and Ca2+[12]. Therefore, our future studies will investigate whether both processes have substantial interconnections in inducing cell death under ${ }^{125}$ I radioactive seeds continuous low dose rate radiation.

Compared with the $\mathrm{X}$-ray single dose radiation, ${ }^{125} \mathrm{I}$ radioactive seeds continuous low dose rate radiation damaged the repopulation of KYSE150 cells which means there are some changes in subcellular structure of KYSE150 cells, such as endoplasmic reticulum and mitochondria. Mitochondria is the main organelles of intracellular ATP production, our results showed intracellular ATP concentration was significantly reduced, suggesting that ${ }^{125}$ I radioactive seeds continuous low dose rate radiation caused damage to mitochondria of KYSE150 cells and the production of ATP was affected. Studies have demonstrated that reduction of ATP can activate AMPK, inhibiting MTOR signal and promoting the autophagy in cells [33]. Endoplasmic reticulum (ER) is a common cellular stress response that is triggered by a variety of conditions that disturb cellular homeostasis, and induces cell apoptosis [34]. Similarly, ionising radiation can activate ER stress signals [35], and radiotherapy has also been shown to induce autophagy in esophageal cancer cells through ER stress [36]. GRP78/Bip1 is an important signal molecule of ER stress, and PERK is one of the downstream signal molecules of ER stress [37]. We found that ER stress signals in esophageal cancer cell line KYSE150 were not significantly activated in the SDR group, while ER stress signals were significantly increased in the early stage after ${ }^{125}$ I radioactive seeds continuous low dose rate radiation, suggesting that ER stress may be involved in autophagy induction of ${ }^{125}$ I radioactive seeds continuous low dose rate radiation on KYSE150 cells. In addition, autophagy is closely related to the apoptosis induced by ER stress. ER and mitochondria are the main sources of free radicals, which play an essential role in the regulation of apoptosis [31][31, 38]. Our results showed that ${ }^{125}$ I-CLDR could activate ER stress signal and damage mitochondrial which caused ROS levels continually to rise which led to apoptosis and autophagy increasing. 
ROS are critical signaling molecules that regulate many signal transduction pathways, and excessive generation of ROS may interfere with cellular signaling pathways and activate subsequent apoptosis and autophagy processes. Therefore, ROS are considered one of the earliest hallmarks of these cell death processes compared to other markers [31]. For instance, ROS have been shown to lead to the induction of the intrinsic pathway of apoptosis by regulating the activity of the Bcl2 family protein via two main mechanisms: direct oxidation of $\mathrm{Bcl} 2$ leading to abrogation of its antiapoptotic activity and increasing the ubiquitination of $\mathrm{Bcl} 2$ [39]. ROS generation has also been shown to regulate Akt-mTOR signaling pathway activity and is subsequently associated with the classical markers of autophagy such as induction of LC3 and conversion of LC3-I to LC3-II [40]. Given that exposure to ${ }^{125}$ I radioactive seeds continuous low dose rate radiation significantly enhanced ROS production in KYSE150 cells, it is possible that the ${ }^{125}$ I radioactive seeds continuous low dose rate radiation triggered apoptosis, autophagy, and downregulated of the Akt-mTOR signaling pathway are related to excessive ROS generation. In addition, ROS can also induce DNA damage [41]. Recent studies have provided mechanistic insight into how ROS can also influence the cellular response to DNA damage. DNA lesions is firstly important to identify the specific lesions responsible for initiating apoptosis before the apoptotic executing pathways can be elucidated [42]. $\mathrm{Y}-\mathrm{H} 2 \mathrm{AX}$ represents the damage of DNA [43], our previous data proved that $\mathrm{Y}-\mathrm{H} 2 \mathrm{AX}$ increased significantly after ${ }^{125} \mathrm{I}$ radioactive seeds continuous low dose rate radiation[44]. DNA-PKロKu70 and Ku80 is a component of the DNA double strand breaks repair apparatus, and cells deficient them are hypersensitive to ionising radiation[45-47]. It suggests that DNA-PK may also have roles in apoptosis $[48,49]$. In sum, it is agree with our results that ${ }^{125}$-CLDR caused ROS levels continually to rise, as a result, more severe DNA damage and impaired the repair ability to KYSE150 cells, which led to apoptosis increasing.

Taken together, our findings confirmed that ${ }^{125}$ I-CLDR could strongly inhibit KYSE1 50 cancer cells. Furthermore, we revealed that autophagy might be an important fate which Akt-mTOR pathway might involve in: ${ }^{125}$ I-CLDR could activate ER stress signal and damage mitochondrial which caused ROS levels continually to rise which led to apoptosis with strong trigger caspase-3 activation, causing decreased esophageal cancer cells Akt-mTOR pathway signaling, which in turn increases autophagy, leading to cell cycle arrest in the G2/M phase and increases radiosensitivity. These findings will be helpful to support the application of esophageal stent loaded with ${ }^{125}$ I seeds in clinic.

\section{Conclusion}

In summary, our study provides a beneficial exploration of biological effects and the potential cellular mechanisms of ${ }^{125}$ I-CLDR on the human esophageal cancer cell line KYSE150. The results showed that 125I-CLDR could strongly inhibit KYSE150 cancer cells compared with SDR. Furthermore, we revealed that cell cycle arrest and apoptosis were not the only fate by ${ }^{125} \mathrm{I}$-CLDR, autophagy might be another important choice which mTOR pathway might be involved in. These findings can support the application of esophageal stent loaded with ${ }^{125}$ I seeds in clinic. Though several questions remained to be addressed, we believe that with development of fundamental research and accumulation of clinical experiences, 
radioactive ${ }^{125}$ I seeds continuous low dose rate radiation can be further transformed and properly used in malignant tumor therapy.

\section{Abbreviations}

\begin{tabular}{|ll|}
\hline Abbreviations & meanings \\
\hline 125 I & iodine 125 \\
\hline 125 I-CLDR & 125 I seeds continuous low dose rate irradiation \\
\hline SDR & single dose irradiation \\
\hline ER & endoplasmic reticulum \\
\hline ROS & Reactive oxygen species \\
\hline DDR & acridine orange \\
\hline
\end{tabular}

\section{Declarations}

Ethical Approval and Consent to participate

Not applicable

\section{Consent for publication}

All authors have reviewed the manuscript and agreed with its contents.

\section{Availability of supporting data}

Not applicable

\section{Competing interests}

All authors of the paper have fulfilled the criteria for authorship; the authors declare there are no conflicts of interest.

\section{Funding}

This work was supported by the National Natural Science Foundation of China (61631001 to J. Wang)

\section{Authors' contributions}

Ping Jiang conceived this manuscript; Junjie Wang conceived the structure of manuscript; Hui Xue and Lifa Du conducted and wrote this manuscript. Hao Wang and Lixiang Xue gave some advices. Chunxiao 
Li revised the language. All authors read and approved the final manuscript.

\section{Acknowledgements}

Not applicable

\section{References}

1. Perisetti $A$, Bellamkonda $M$, Konda $M$, et al. Tumor-associated antigens and their antibodies in the screening, diagnosis, and monitoring of esophageal cancers. Eur J Gastroenterol Hepatol 2020

2. Schizas D, Charalampakis N, Kole C, et al. Immunotherapy for esophageal cancer: a 2019 update. IMMUNOTHERAPY-UK 2020;12:203-218

3. van der Bogt RD, Vermeulen BD, Reijm AN, Siersema PD, Spaander M. Palliation of dysphagia. Best Pract Res Clin Gastroenterol 2018;36-37:97-103

4. Sunde B, Johnsen G, Jacobsen AB, et al. Effects of neoadjuvant chemoradiotherapy vs chemotherapy alone on the relief of dysphagia in esophageal cancer patients: secondary endpoint analysis in a randomized trial. DIS ESOPHAGUS 2019;32

5. Guo JH, Teng GJ, Zhu GY, et al. Self-expandable esophageal stent loaded with 125 I seeds: initial experience in patients with advanced esophageal cancer. RADIOLOGY 2008;247:574-581

6. Takeuchi M, Tanikawa M, Nagasaka K, et al. Anti-Tumor Effect of Inhibition of DNA Damage Response Proteins, ATM and ATR, in Endometrial Cancer Cells. Cancers (Basel) 2019;11

7. Xia F, Powell SN. The molecular basis of radiosensitivity and chemosensitivity in the treatment of breast cancer. SEMIN RADIAT ONCOL 2002;12:296-304

8. Wang $\mathrm{H}$, Jiang $\mathrm{H}$, Corbet $\mathrm{C}$, et al. Piperlongumine increases sensitivity of colorectal cancer cells to radiation: Involvement of ROS production via dual inhibition of glutathione and thioredoxin systems. CANCER LETT 2019;450:42-52

9. Li L, Liu WL, Su L, Lu ZC, He XS. The Role of Autophagy in Cancer Radiotherapy. CURR MOL PHARMACOL 2020;13:31-40

10. He Q, Li J, Dong F, Cai C, Zou X. LKB1 promotes radioresistance in esophageal cancer cells exposed to radiation, by suppression of apoptosis and activation of autophagy via the AMPK pathway. MOL MED REP 2017;16:2205-2210

11. Ma S, Miao H, Luo Y, et al. FePt/GO Nanosheets Suppress Proliferation, Enhance Radiosensitization and Induce Autophagy of Human Non-Small Cell Lung Cancer Cells. INT J BIOL SCI 2019;15:9991009

12. Song S, Tan J, Miao Y, Li M, Zhang Q. Crosstalk of autophagy and apoptosis: Involvement of the dual role of autophagy under ER stress. J CELL PHYSIOL 2017;232:2977-2984

13. Wang H, Li J, Qu A, et al. The different biological effects of single, fractionated and continuous low dose rate irradiation on CL187 colorectal cancer cells. RADIAT ONCOL 2013;8:196 
14. Wang J, Wang J, Liao A, Zhuang H, Zhao Y. The direct biologic effects of radioactive $125 \mathrm{I}$ seeds on pancreatic cancer cells PANC-1, at continuous low-dose rates. Cancer Biother Radiopharm 2009;24:409-416

15. Liao A, Wang J, Wang J, Zhuang $H$, Zhao Y. Relative biological effectiveness and cell-killing efficacy of continuous low-dose-rate 125 l seeds on prostate carcinoma cells in vitro. INTEGR CANCER THER 2010;9:59-65

16. Zhuang HQ, Wang JJ, Liao AY, Wang JD, Zhao Y. The biological effect of 125 I seed continuous low dose rate irradiation in CL187 cells. J Exp Clin Cancer Res 2009;28:12

17. Paglin $S$, Hollister $T$, Delohery $T$, et al. A novel response of cancer cells to radiation involves autophagy and formation of acidic vesicles. CANCER RES 2001;61:439-444

18. Qin J, Zhu HD, Guo JH, et al. Factors associated with overall survival and relief of dysphagia in advanced esophageal cancer patients after $125 \mathrm{I}$ seed-loaded stent placement: a multicenter retrospective analysis. DIS ESOPHAGUS 2019;32

19. Gongpan P, Lu Y, Wang F, Xu Y, Xiong W. AS160 controls eukaryotic cell cycle and proliferation by regulating the CDK inhibitor p21. CELL CYCLE 2016;15:1733-1741

20. Petrachkova T, Wortinger LA, Bard AJ, et al. Lack of Cyclin B1 in zebrafish causes lengthening of $G 2$ and M phases. DEV BIOL 2019;451:167-179

21. Ahn JH, Jang GH, Lee M. Defective autophagy in multidrug resistant cells may lead to growth inhibition by BH3-mimetic gossypol. J CELL PHYSIOL 2013;228:1496-1505

22. Oh $\mathrm{HN}$, Oh KB, Lee MH, et al. JAK2 regulation by licochalcone $\mathrm{H}$ inhibits the cell growth and induces apoptosis in oral squamous cell carcinoma. PHYTOMEDICINE 2019;52:60-69

23. Razaghi A, Heimann K, Schaeffer PM, Gibson SB. Negative regulators of cell death pathways in cancer: perspective on biomarkers and targeted therapies. APOPTOSIS 2018;23:93-112

24. Xie W, Jiao B, Bai Q, et al. Chemoptogenetic ablation of neuronal mitochondria in vivo with spatiotemporal precision and controllable severity. ELIFE 2020;9

25. Sivakumar D, Sivaraman T. A Review on Structures and Functions of Bcl-2 Family Proteins from Homo sapiens. Protein Pept Lett 2016;23:932-941

26. Feng FB, Qiu HY. Effects of Artesunate on chondrocyte proliferation, apoptosis and autophagy through the PI3K/AKT/mTOR signaling pathway in rat models with rheumatoid arthritis. BIOMED PHARMACOTHER 2018;102:1209-1220

27. Li Z, Dong H, Li M, et al. Honokiol induces autophagy and apoptosis of osteosarcoma through $\mathrm{PI3K} / \mathrm{Akt} / \mathrm{mTOR}$ signaling pathway. MOL MED REP 2018;17:2719-2723

28. Zhou M, Shen S, Zhao X, Gong X. Luteoloside induces G0/G1 arrest and pro-death autophagy through the ROS-mediated AKT/mTOR/p70S6K signalling pathway in human non-small cell lung cancer cell lines. Biochem Biophys Res Commun 2017;494:263-269

29. Zhang H, Zhang X, Zhang J. MiR-129-5p inhibits autophagy and apoptosis of H9c2 cells induced by hydrogen peroxide via the PI3K/AKT/mTOR signaling pathway by targeting ATG14. Biochem 
Biophys Res Commun 2018;506:272-277

30. Kaminskyy VO, Zhivotovsky B. Free radicals in cross talk between autophagy and apoptosis. Antioxid Redox Signal 2014;21:86-102

31. Li Y, Guo Y, Fan Y, et al. Melatonin Enhances Autophagy and Reduces Apoptosis to Promote Locomotor Recovery in Spinal Cord Injury via the PI3K/AKT/mTOR Signaling Pathway. NEUROCHEM RES 2019;44:2007-2019

32. Russell RC, Yuan HX, Guan KL. Autophagy regulation by nutrient signaling. CELL RES 2014;24:42-57

33. Yang Y, Wang G, Wu W, et al. Camalexin Induces Apoptosis via the ROS-ER Stress-Mitochondrial Apoptosis Pathway in AML Cells. OXID MED CELL LONGEV 2018;2018:7426950

34. Zhang B, Wang Y, Pang X, et al. ER stress induced by ionising radiation in IEC-6 cells. INT J RADIAT BIOL 2010;86:429-435

35. Pang XL, He G, Liu YB, Wang Y, Zhang B. Endoplasmic reticulum stress sensitizes human esophageal cancer cell to radiation. World J Gastroenterol 2013;19:1736-1748

36. Yoshida H. ER stress and diseases. FEBS J 2007;274:630-658

37. Huang ML, Chiang S, Kalinowski DS, et al. The Role of the Antioxidant Response in Mitochondrial Dysfunction in Degenerative Diseases: Cross-Talk between Antioxidant Defense, Autophagy, and Apoptosis. OXID MED CELL LONGEV 2019;2019:6392763

38. Galadari S, Rahman A, Pallichankandy S, Thayyullathil F. Reactive oxygen species and cancer paradox: To promote or to suppress? Free Radic Biol Med 2017;104:144-164

39. Zhao GX, Pan H, Ouyang DY, He XH. The critical molecular interconnections in regulating apoptosis and autophagy. ANN MED 2015;47:305-315

40. Srinivas US, Tan B, Vellayappan BA, Jeyasekharan AD. ROS and the DNA damage response in cancer. REDOX BIOL 2019;25:101084

41. Roos WP, Kaina B. DNA damage-induced cell death: from specific DNA lesions to the DNA damage response and apoptosis. CANCER LETT 2013;332:237-248

42. Kariolis MS, Miao YR, Diep A, et al. Inhibition of the GAS6/AXL pathway augments the efficacy of chemotherapies. J CLIN INVEST 2017;127:183-198

43. Du Lifa, liu jingjia, et al. Inhibitory effect and its mechanism of $125 \mathrm{I}$ seeds by continuous low-dose irradiation on human esophageal cancer cell line KYSE150. Chin J Radiol Med Prot 2014;34:415-418

44. Muller C, Rodrigo G, Calsou P, Salles B. [DNA-dependent protein kinase: a major protein involved in the cellular response to ionizing radiation]. Bull Cancer 1999;86:977-983

45. Corrigendum to: Genetic interaction between DNA repair factors PAXX, XLF, XRCC4 and DNA-PKcs in human cells. FEBS OPEN BIO 2020

46. Xing $M$, Oksenych V. Genetic interaction between DNA repair factors PAXX, XLF, XRCC4 and DNAPKcs in human cells. FEBS OPEN BIO 2019;9:1315-1326

47. Sand-Dejmek J, Adelmant G, Sobhian B, et al. Concordant and opposite roles of DNA-PK and the "facilitator of chromatin transcription" (FACT) in DNA repair, apoptosis and necrosis after cisplatin. 
MOL CANCER 2011;10:74

48. Ho SR, Mahanic CS, Lee YJ, Lin WC. RNF144A, an E3 ubiquitin ligase for DNA-PKcs, promotes apoptosis during DNA damage. Proc Natl Acad Sci U S A 2014;111:E2646-E2655

Figures

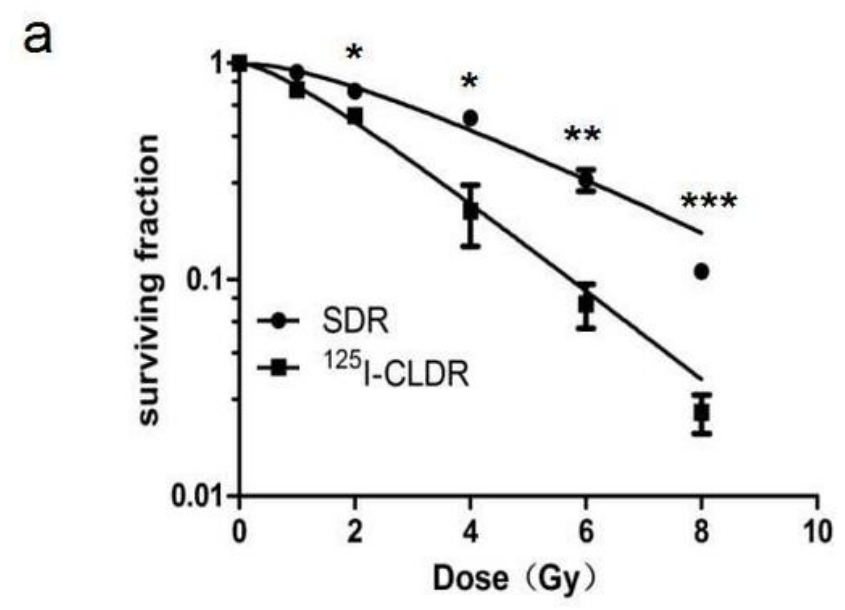

b

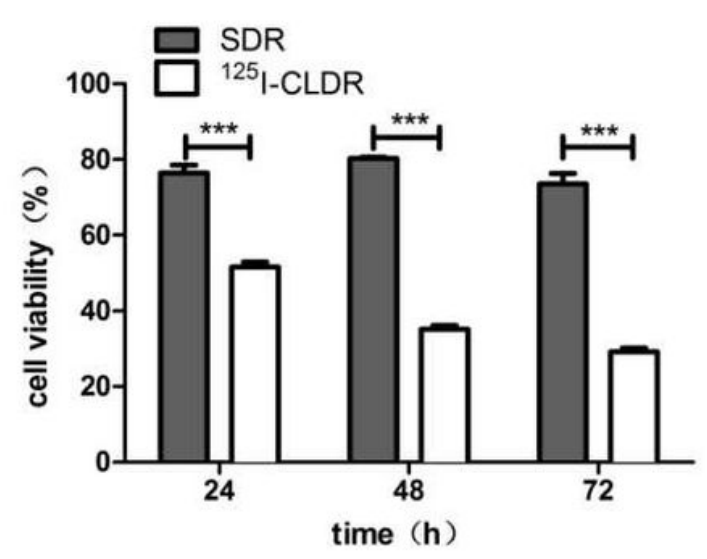

C

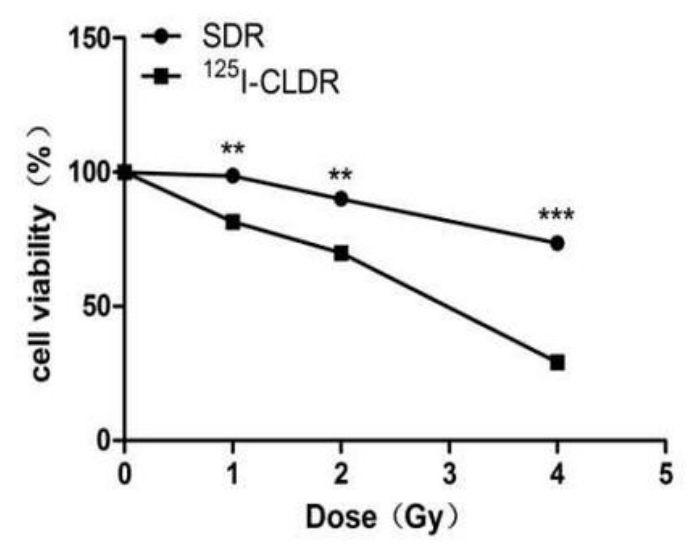

Figure 1

KYSE150 cells were more radiosensitive to 125I-CLDR than SDR. a. Cell dose survival curves after two types of irradiation. b. Cell activity in each group at different time points after 4Gy irradiation. c. Cell activity in each irradiation group after $72 \mathrm{~h}$ of different irradiation doses. ${ }^{*} \mathrm{P}<0.05$; $* * \mathrm{P}<0.01 ; * * * \mathrm{P}<$ 0.001 . 
b
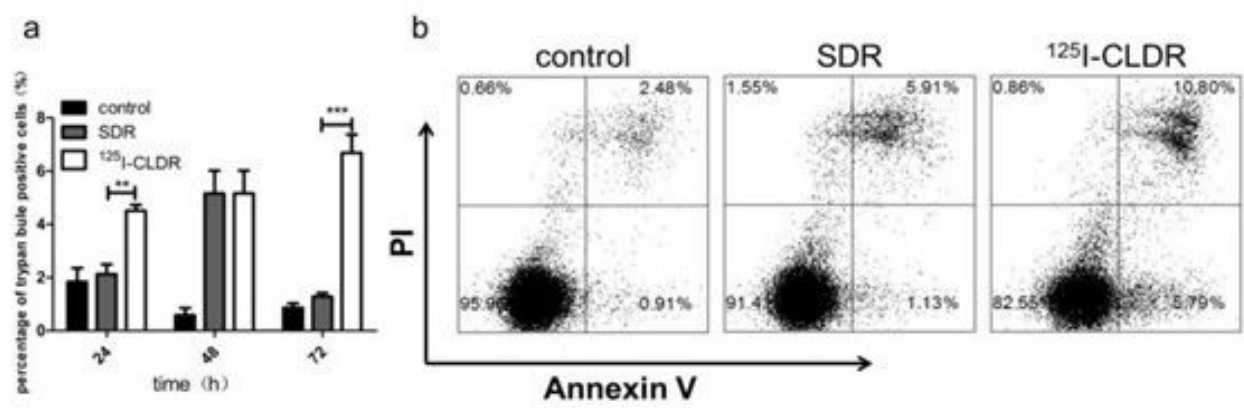

Annexin V
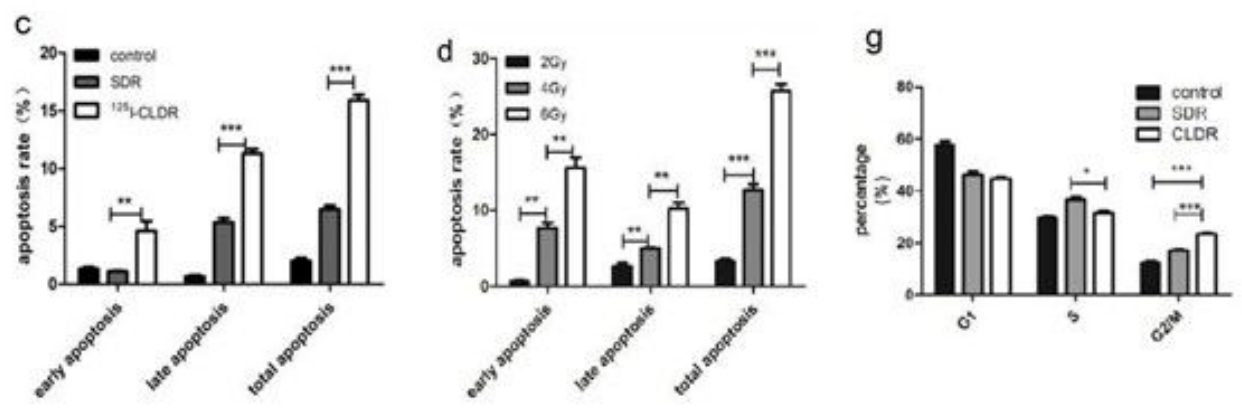

f

control

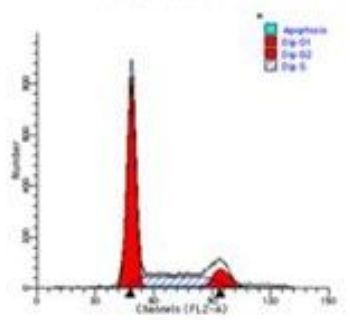

SDR

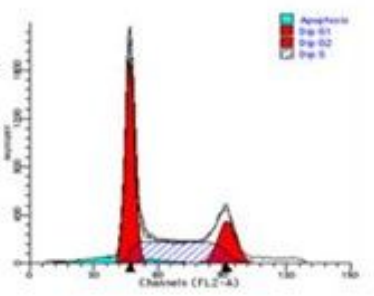

125|-CLDR

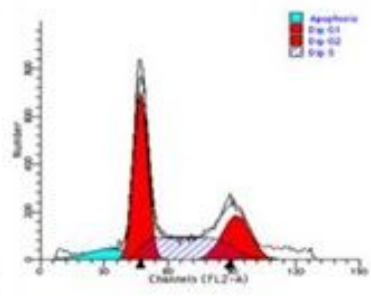

e
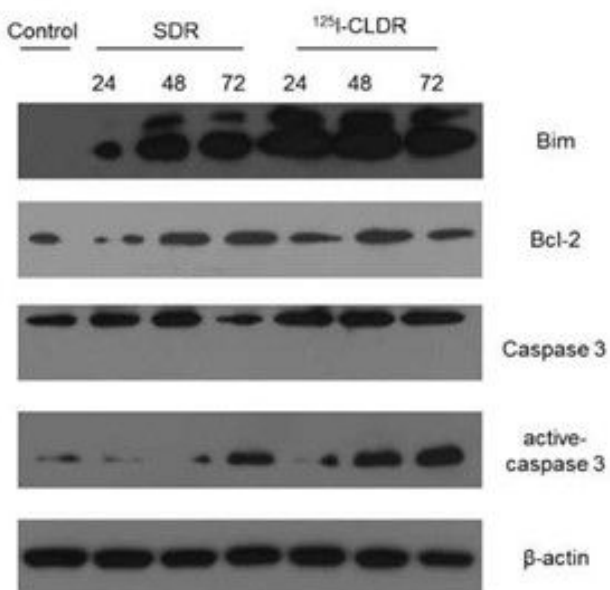

$\beta$-actin

\section{Figure 2}

It is showed more proportions of the early and late apoptosis rate as well as cells at G2/M phase in 125ICLDR group. a. Percentage of placenta blue staining of KYSE150 cells at different time points after 4Gy irradiation. b. flow analysis of apoptosis in each group at $72 \mathrm{~h}$ after $4 \mathrm{~Gy}$ irradiation. c. Histogram of the statistical results of analysis rate of each irradiation group at $72 \mathrm{~h}$ after $4 \mathrm{~Gy}$ irradiation. $d$. Histogram of the statistical results of changes of analysis rate of each irradiation group at $72 \mathrm{~h}$ after different irradiation doses. e. Expression of apoptosis-related proteins after irradiation. f. Flow analysis of cell cycle distribution in each group $72 \mathrm{~h}$ after $4 \mathrm{~Gy}$ irradiation. g. Histogram of flow analysis of cell cycle distribution in each group at $72 \mathrm{~h}$ after $4 \mathrm{~Gy}$ irradiation. $\mathrm{h}$. The expression of CyclinB1 in protein level after irradiation. * $\mathrm{P}<0.05 ; * * \mathrm{P}<0.01 ; * * * \mathrm{P}<0.001$. 
a

Control

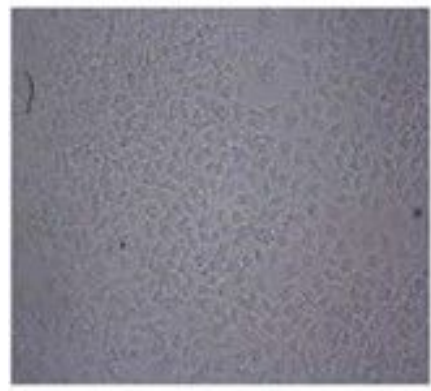

SDR

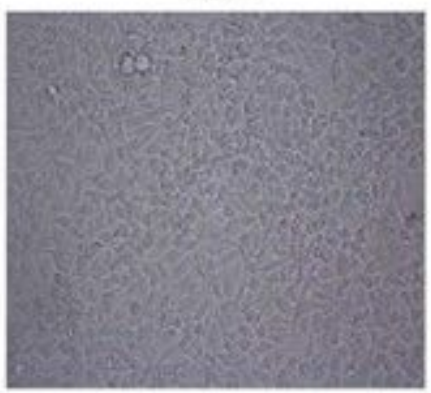

125I-CLDR

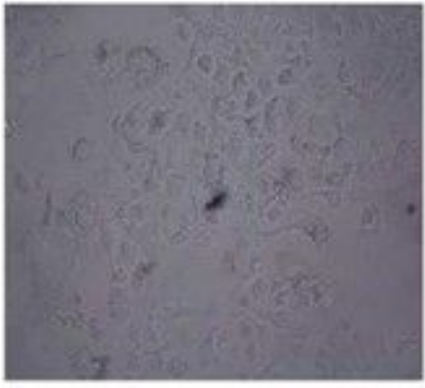

C

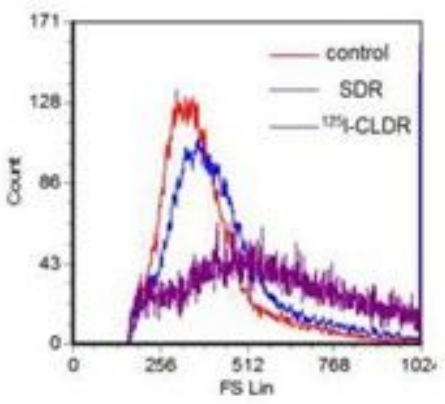

d

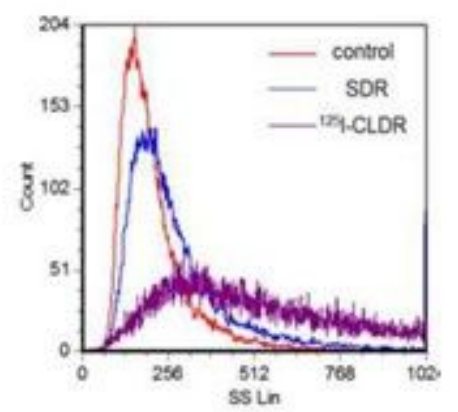

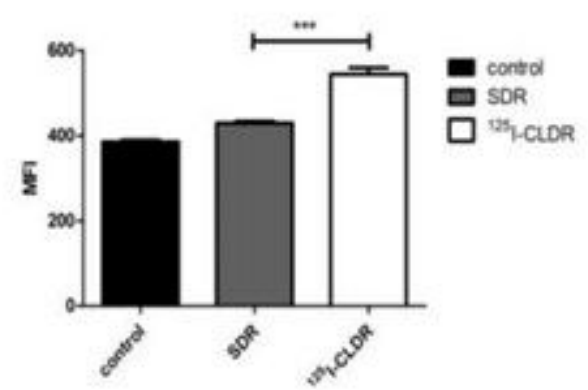

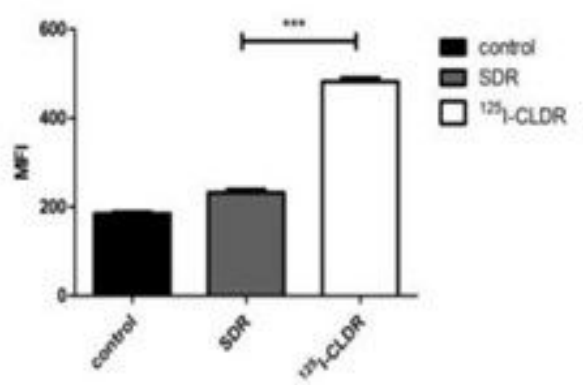

b
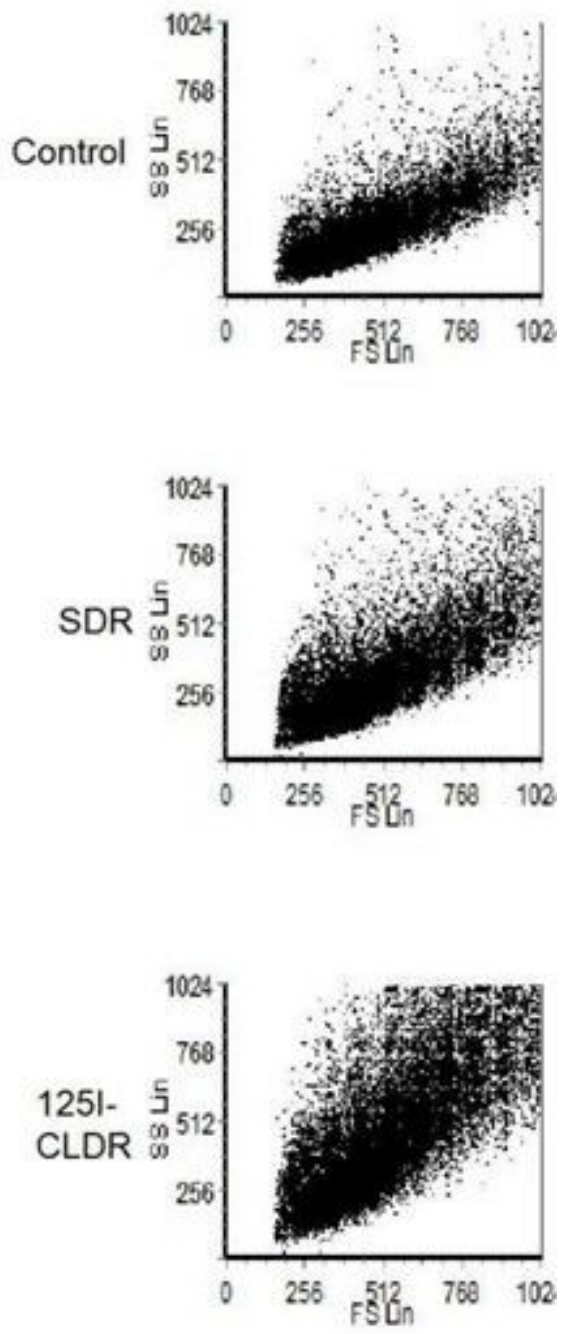

\section{Figure 3}

Cells became bigger and grainy in 125I-CLDR group. a. Cell morphology of each group at $72 \mathrm{~h}$ after 4Gy irradiation by inverted phase contrast microscope (20x). b. Cytometry detection of FS and SS in each group at $72 \mathrm{~h}$ after $4 \mathrm{~Gy}$ irradiation. c. Cell size after irradiation. $\mathrm{d}$. Cell granularity after irradiation. $* * * \mathrm{P}<$ 0.001 . 
a
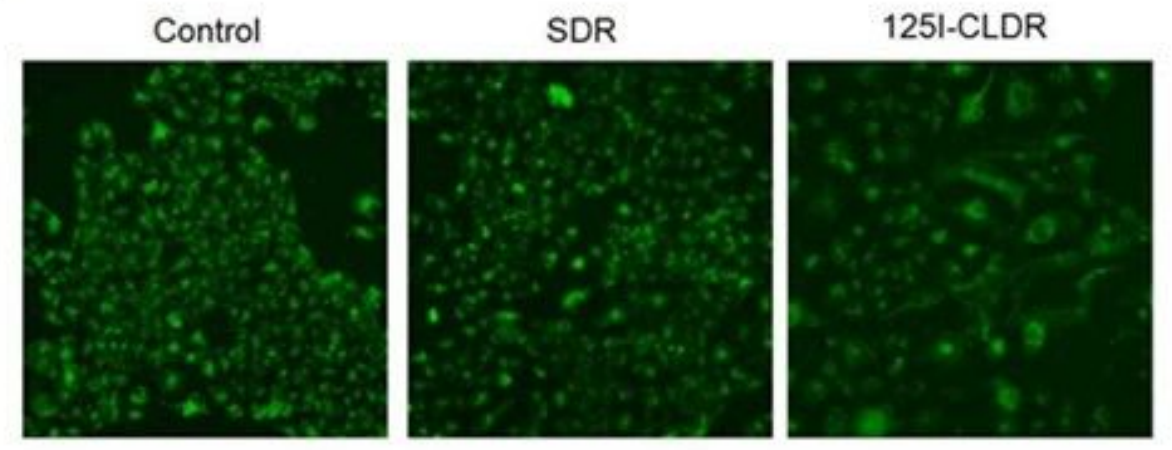

b

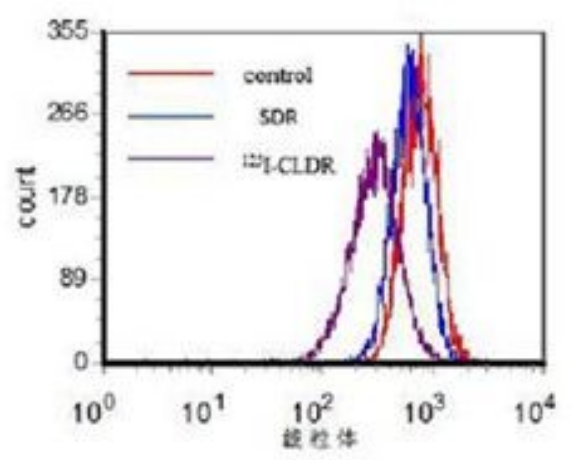

C

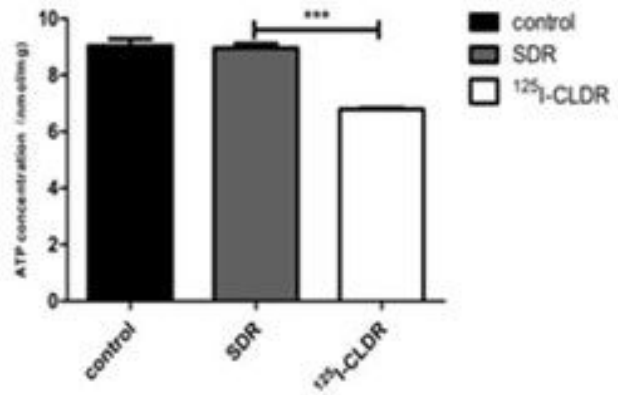

d

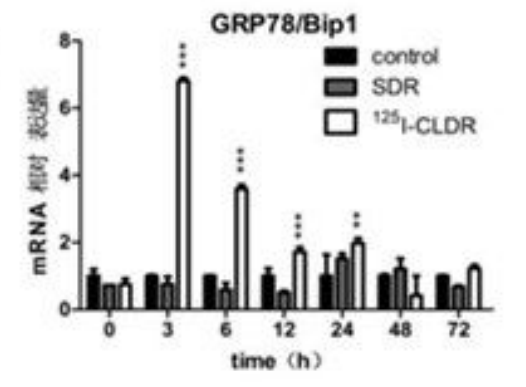

e

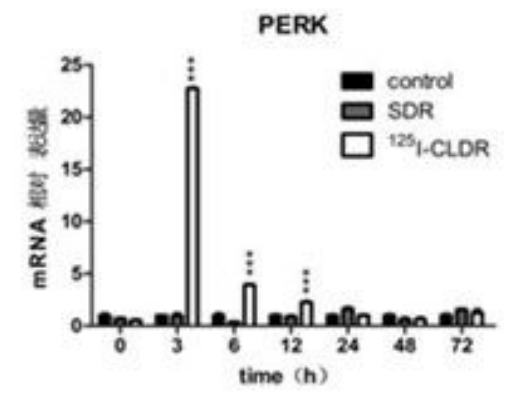

\section{Figure 4}

ER stress signal was elevated and ATP concentration was decreased. a. Mitochondrial fluorescence intensity in each experimental group at $24 \mathrm{~h}$ after $4 \mathrm{~Gy}$ irradiation. b. MFI of mitochondria in each group at 24h after 4Gy irradiation. c. intracellular ATP concentration at 24h after 4Gy irradiation. d. The mRNA level of GRP78/Bip1 after 4Gy irradiation. e. The mRNA level of PERK after 4Gy irradiation. * $\mathrm{P}<0.01$; * * $\star P<0.001$. 
a

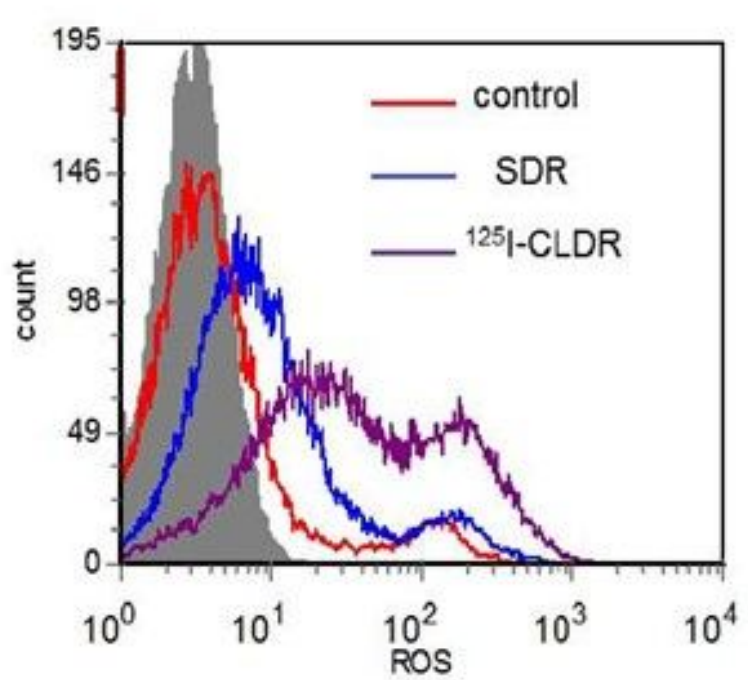

b

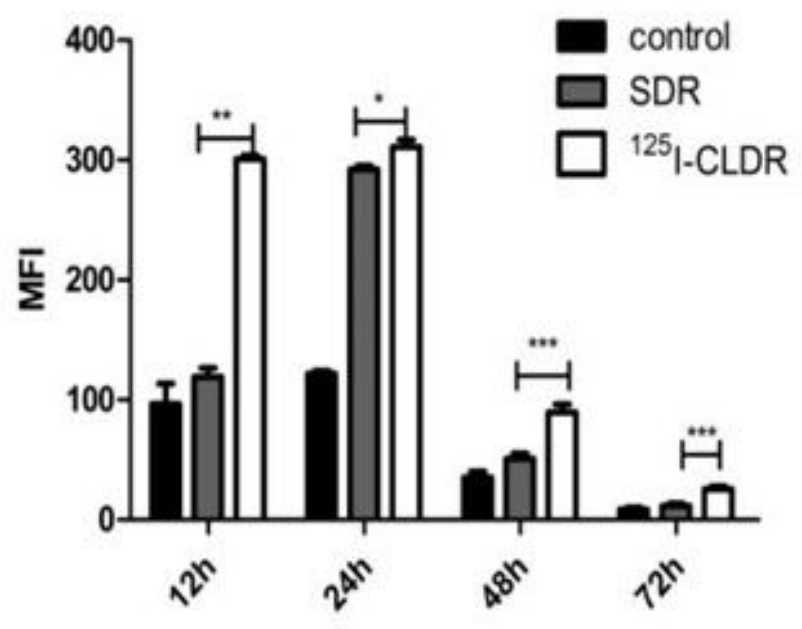

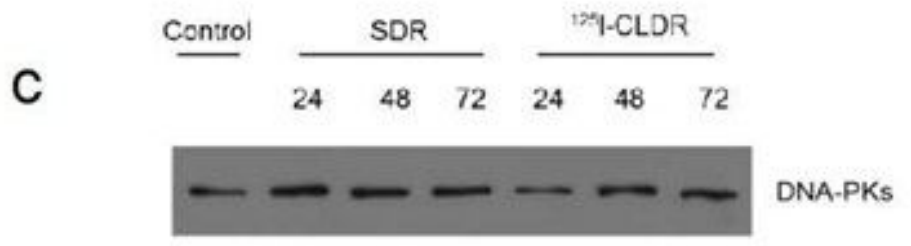
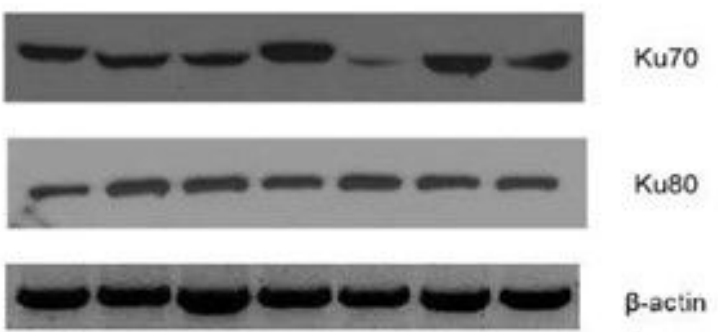

Figure 5

ROS levels was elevated and DNA damage was aggravated. a. ROS levels in each group at 72h after 4Gy irradiation. b.ROS levels in each group at different time points after 4Gy irradiation. c. Expression of DNApks, Ku70 and Ku80 in protein level after irradiation. ${ }^{*} \mathrm{P}<0.05 ; * * \mathrm{P}<0.01 ; * * * \mathrm{P}<0.001$. 
a
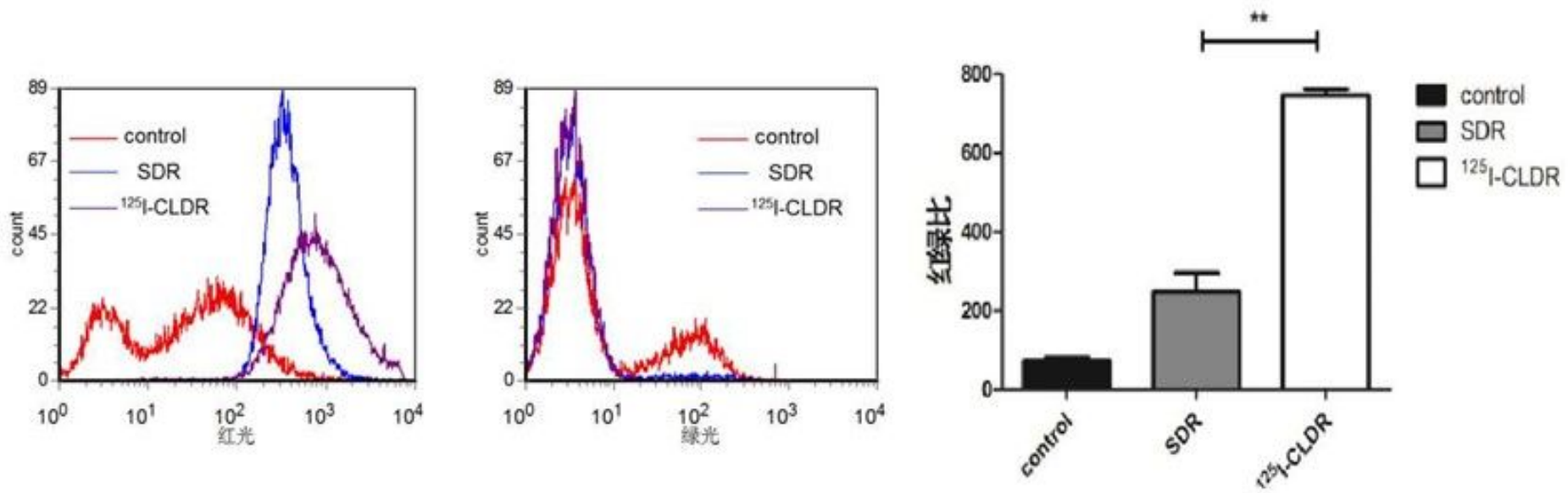

b
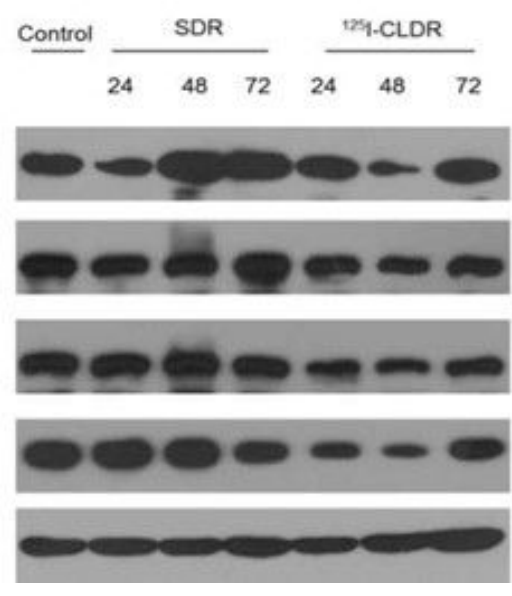

P-AKT

mTOR

p-mTOR

p-S6

B-actin

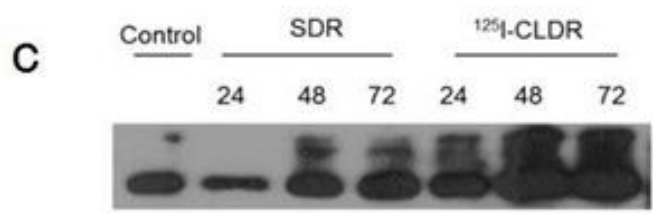

LC3-1I

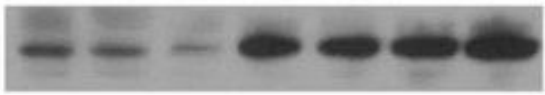

ATG5

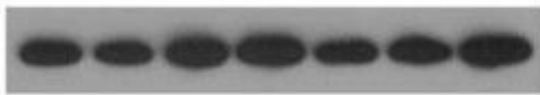

Beclin1

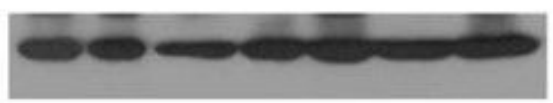

$\beta$-actin

\section{Figure 6}

Activation of Akt-mTOR signaling pathways was decreased and autophagy was increased in 125I-CLDR group. a. AO staining result showed that red: green ratio increased in 125I-CLDR group after irradiation. b. The expression of p-Akt, p-mTOR and p-S6 in protein level after irradiation. c. The expression of LC3-II, ATG5 and Beclin 1 in protein level after irradiation. ${ }^{*} \mathrm{P}<0.01$. 